\title{
Antibiotic Prophylaxis with Quinolones in Afebrile Neutropenic Patients: Is there Enough Evidence for Mortality Effect?
}

\author{
Gláucia Fernandes $\operatorname{Cota}^{*}{ }^{*, 2,3}$, Tatiani Fereguetti ${ }^{2}$ and Marcos Roberto de Sousa ${ }^{1,4}$ \\ ${ }^{I}$ Clinics Hospital from Federal University of Minas Gerais - UFMG, Brazil \\ ${ }^{2}$ Eduardo de Menezes Hospital, Fundação Hospitalar do Estado de Minas Gerais - FHEMIG, Brazil \\ ${ }^{3}$ Post Graduation Program of Health Sciences at Rene Rachou Institute - Fundação Oswaldo Cruz (FIOCRUZ), Brazil \\ ${ }^{4}$ Post Graduation Program in Adult Health Sciences at Medical School from Federal University of Minas Gerais - \\ UFMG, Belo Horizonte, Minas Gerais, Brazil
}

\begin{abstract}
Febrile neutropenia causes significant morbidity and mortality in patients receiving antineoplastic chemotherapy. During the last decade, some trials have shown reduction in febrile episodes and bacteraemia with antibiotic prophylaxis. On the other hand, the emergence of fluoroquinolone-resistant bacteria in units practicing quinolones prophylaxis has been described and there is justified concern about the implications of this fact. Recent published guidelines now recommend quinolones prophylaxis for patients with neutropenia expected to last as least 7 days. They are based in a meta-analysis of placebo-controlled or no treatment-controlled trials of quinolone prophylaxis, which demonstrated risk reduction in mortality among drug treated recipients. In contrast, previous meta-analyses and the two major trials with two thirds of the number of patients of the largest meta-analysis failed to show this survival advantage. Using two sensitive tests, Egger's and Trim and Fill methods, we found a "small study effect" which acts exaggerating and making the result positive when they are probably neutral. In spite of the considerable number of trials and reviews involving prophylactic antibiotics for neutropenic patients, available evidence is hypothesis generator but not sufficient to state there is mortality benefit.
\end{abstract}

Keywords: Antibiotic prophylaxis, mortality, neutropenia, quinolones.

\section{BACKGROUND}

Infection is the most common and fatal complication associated with neutropenia and the appearance of fever is the first manifestation of a potentially life threatening infection. There are many studies on using antibiotics to prevent infection during chemotherapy. Seven meta-analyses were conducted to assess the efficacy of antibiotics including quinolones for preventing bacterial infections in neutropenic patients. Four meta-analyses published from 1990 to 2005 concluded that quinolones prophylaxis reduces various infection-related outcomes, but not mortality [1-4]. In 2005, two large multi-centric randomized double-blind placebocontrolled trials evaluating levofloxacin prophylaxis were published $[5,6]$. In both studies the reduction in mortality did not reach statistical significance. Three new metaanalyses [7-9], including more studies and the above mentioned large trials suggested that quinolones prophylaxis could reduce mortality. Current guidelines recommend now that antibiotic prophylaxis is considered in all patients at high and intermediate risk of febrile neutropenia $[10,11]$. This includes patients undergoing allogeneic hematopoietic cell transplantation (HCT) and patients undergoing induction therapy for acute leukemia.

Address correspondence to this author at the Avenida do Contorno, 9636/1607, Belo Horizonte, ZIP-CODE 30110-068, MG, Brazil; Tel/Fax: + 5531 3295-6695; E-mail: glauciacota@uol.com.br
This paper is a critical appraisal of available evidence, exploring the influence of the methodological differences and the "small study effect" (publication bias) on the results of systematic reviews addressing quinolones prophylaxis for afebrile neutropenic patients.

\section{WHAT IS THE AVAILABLE EVIDENCE?}

Main design characteristics of the published metaanalyses assessing antibiotic prophylaxis for afebrile neutropenic patients are presented in Table $\mathbf{1}$.

The three meta-analyses conducted in the decade of 1990 [1-3] included randomized controlled trials using a prophylactic regimen with only quinolones or a combination of quinolones and antibiotics which are active against Grampositive pathogens. These reviews showed a significant reduction of Gram-negative bacteraemia with quinolonesbased regimens but failed to show a reduction in infectionrelated mortality with prophylaxis.

Van de Watering et al., [4] included randomized trials published until 2002 that compared oral based prophylactic antibiotics. The overall analysis that included the thirteen trials using quinolones or trimethoprim-sulfamethoxazole (TMP/SMZ) prophylaxis showed a significant reduction in infection related mortality with prophylaxis $(\mathrm{OR}=0.56$; $95 \%$ CI 0.34 to $0.96 ; \mathrm{I}^{2}=0 \%$ ). Separate ORs for TMP/SMZ and quinolones trials did not reach statistical significance and the authors did not evaluate overall mortality. 
Table 1. Main Characteristics and Results of Meta-Analyses

\begin{tabular}{|c|c|c|c|c|c|}
\hline Study & $\begin{array}{c}\text { n for } \\
\text { Mortality }\end{array}$ & $\begin{array}{c}\text { Study Quality } \\
\text { Assessment }\end{array}$ & $\begin{array}{c}\text { Publication Bias } \\
\text { Evaluation }\end{array}$ & $\begin{array}{c}\text { Heterogeneity } \\
\text { Assessment }\end{array}$ & Results and Commentaries \\
\hline \hline Cruciani et al., 1996 & 205 & Performed & Fail-Safe N & Adequate & $\begin{array}{c}\text { Antibiotic prophylaxis had no effect in infection related or } \\
\text { all-cause mortality }\end{array}$ \\
\hline Rotstein et al., 1997 & 384 & Performed & Not presented & Not presented & Antibiotic prophylaxis had no effect in all-cause mortality \\
\hline Engels et al., 1998 & 406 & Performed & Not presented & Not presented & Antibiotic prophylaxis had no effect in all-cause mortality \\
\hline $\begin{array}{c}\text { Van de Wetering } \text { et al., } \\
\text { 2005 }\end{array}$ & 966 & Performed & Not presented & Adequate & Antibiotic prophylaxis reduced infection related mortality \\
\hline Gafter-Gvili et al., 2005 & 1244 & Performed & Tests not presented & Adequate & Antibiotic prophylaxis reduced all-cause mortality \\
\hline Leibovici et al., 2006 & 3360 & Performed & Tests not presented & Adequate & Antibiotic prophylaxis reduced all-cause mortality \\
\hline Imran et al., 2008 & 2719 & Performed & Not presented & Adequate & $\begin{array}{c}\text { The reduction in all-cause mortality did not reach } \\
\text { statistical significance }\end{array}$ \\
\hline
\end{tabular}

Gafter-Gvili et al., [12] have used a more comprehensive inclusion criteria and considered studies comparing different types of antibiotic therapy with placebo, no intervention, or with another antibiotic, for the prophylaxis of bacterial infections in afebrile neutropenic patients. In addition, they included trials published up to the 2000s and randomized controlled trials (RTC) or quasi-RCTs (in which allocation generation was inadequate, such as date of birth, case record number, etc.). The authors concluded that fluoroquinolone prophylaxis reduced the risk for all-cause mortality (relative risk, 0.52 [CI, 0.35 to 0.77$]$ ), as well as infection-related mortality (relative risk 0.38 [CI, 0.21 to 0.69$]$ ). However, one study in which was used nalidixic acid, not a true quinolone, and other three trials studying prophylaxis strategies that combine quinolones plus antibiotics with coverage against Gram-positive pathogens were included.

The meta-analysis done by Gafter-Gvili et al., was updated by Leibovici et al., in 2006 [7] with data from two randomized trials published in 2005. The authors observed reduction in all-cause mortality (RR $0.67 ; 95 \% \mathrm{CI} 0.48$ to 0.93 ). This is the most comprehensive review including the highest number of studies and patients. They also included patients using prophylaxis with other antibiotics associated with quinolones.

Imran et al., [8] performed a meta-analysis with more stringent criteria and only randomized, blinded, placebocontrolled trials of fluoroquinolone prophylaxis in neutropenic patients were considered. They excluded studies that used quinolones prophylaxis plus other antibiotic with coverage for positive Gram cocci. Using these criteria the sample size was reduced, and the confidence intervals grew in proportion. Although the authors concluded that: "prophylaxis with a fluoroquinolone is associated with a favorable effect involving a reduction in overall mortality of these patients", their results did not reach statistical significance (RR 0.76; 95\%CI 0.54-1.08).

\section{SINCE THE TWO LARGE STUDIES DID NOT FIND MORTALITY BENEFIT, ARE THE SMALL STUDIES RESPONSIBLE FOR POSITIVE RESULTS?}

Reporting biases arise when the dissemination of research findings is influenced by the nature and direction of results. Publication bias occurs when statistically significant "positive" studies are more likely to be published, published rapidly, published in English, published more than once, published in high impact journals, and cited by others [13]. Such studies are therefore also more likely to be identified and included in systematic reviews, which may introduce bias [14]. Low methodological quality of studies included in a systematic review is another important source of bias [15]. By selectively failing to make public the negative results of clinical interventions, publication bias has the potential to lead to an erroneous collective conclusion that an ineffective therapy is effective or to amplify the apparent beneficial effect of an effective therapy [16]. All these biases are more likely to affect small studies than large ones. The smaller a study the larger the treatment effect necessary for the results to be significant. Bias in a systematic review may therefore become evident through an association between the size of the treatment effect and study size - such associations may be examined both graphically and statistically.

Two meta-analyses $[7,12]$ found a reduction in mortality with the use of quinolones prophylaxis. In fact, one [7] is an update from the other [12]. We analyzed their data [17] using Egger's test of the Intercept and 'Trim and Fill' test. Both tests suggested the presence of "small study effects" (the tendency for the smaller studies in a meta-analysis to show larger treatment effects).

Funnel plots are simple scatter plots of the treatment effects estimated from individual studies (horizontal axis) against some measure (usually inverse variance) of study size (vertical axis). Because precision in estimating the underlying treatment effect increases as a study's sample size increases, effect estimates from small studies scatter more widely at the bottom of the graph, with the spread narrowing among larger studies. The funnel plot is based on the fact that precision in estimating the underlying treatment effect will increase as the sample size of component studies increases. Results from small studies will scatter widely at the bottom of the graph, with the spread narrowing among larger studies. In the absence of bias, the plot will resemble a symmetrical inverted funnel. Conversely, if there is bias, funnel plots will often be skewed and asymmetrical [18]. Duval and Tweedie have proposed "trim and fill"; a method based on adding studies to a funnel plot so that it becomes symmetrical [16]. Smaller studies are omitted until the funnel plot is symmetrical (trimming). The trimmed funnel plot is used to estimate the true "centre" of the funnel, and 


\section{Funnel Plot of Precision by Log risk ratio}

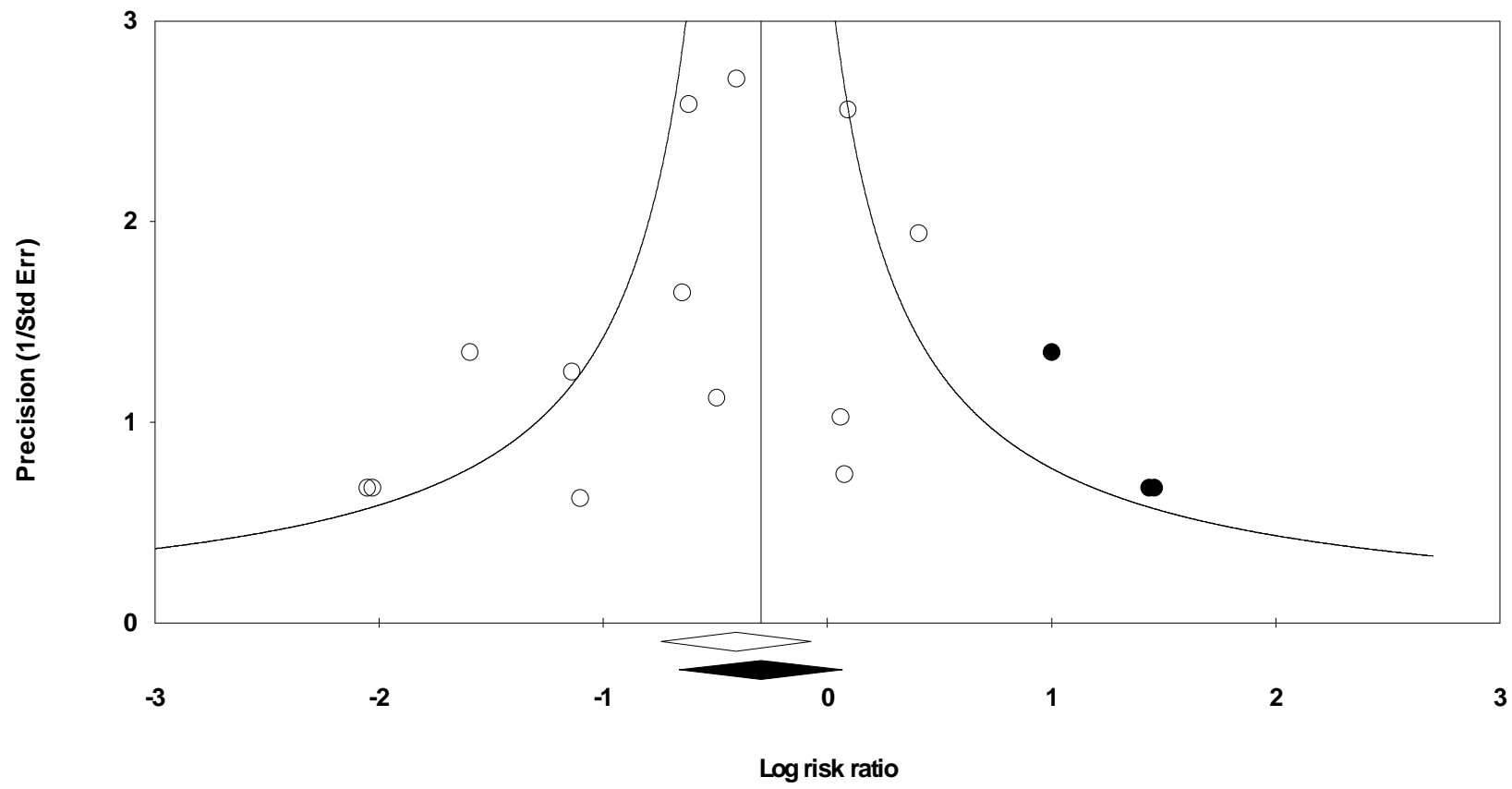

Fig. (1). Funnel plot with imputed studies (arrows) suggests presence of publication bias which acts exaggerating and making the result positive when they are probably neutral. Trim and fill method suggests that 3 studies are missing in the meta-analysis of Leibovici et al., 2006.

then the omitted studies and their missing "counterparts" around the centre are replaced (filling). It provides an estimate of the number of missing studies and an adjusted treatment effect, including the "filled" studies (Fig. 1).

Egger suggests that we assess publication bias by using precision (the inverse of the standard error) to predict the standardized effect (effect size divided by the standard error). In this equation, the size of the treatment effect is captured by the slope of the regression line (B1) while bias is captured by the intercept (B0) [19]. Although Egger's test is a more powerful tool to evaluate asymmetry in metaanalyses with continuous than dichotomous outcomes [20], its application is adequate in analysis including 10 or more studies. This approach may offer a number of advantages over the rank correlation approach. Under some circumstances this may be a more powerful test. Additionally, this approach can be extended to include more than one predictor variable, which means that we can simultaneously assess the impact of several factors, including sample size, on the treatment effect. In the metaanalysis done in 2005 [12], the intercept (B0) is $-1.65,95 \%$ confidence interval $(-3.00$ to -0.30$)$, with $\mathrm{t}=2.72, \mathrm{df}=10$. The 1 -tailed p-value (recommended) is 0.01 . In the meta-analysis done in 2006 [7], the intercept (B0) is $-0.90,95 \%$ confidence interval $(-2.09$ to 0.30$)$, with $\mathrm{t}=1.65546, \mathrm{df}=11$. The 1 -tailed p-value (recommended) is 0.06 . These results suggested publication bias.

Results of 'Trim and Fill' test [19] also suggest that results are probably exaggerated by "small study effect". In the meta-analysis of Gafter-Gvili et al., under the randomeffects model the point estimate ( $95 \%$ confidence interval) for the combined studies is $0.56(0.33$ to 0.96$)$. Using Trim and Fill the imputed point estimate is 0.92 ( 0.49 to 1.72$)$. In the meta-analysis of Leibovici et al., under the randomeffects model the point estimate ( $95 \%$ confidence interval) for the combined studies is 0.67 (0.48 to 0.93$)$. Using Trim and Fill the imputed point estimate is 0.74 (0.52 to 1.07). In both cases, imputed point estimate have confidence intervals passing through 1, suggesting null effect transformed in positive effect by small studies. In Leibovici et al., work, fixed effect model was used throughout the review, except in the event of significant heterogeneity between the trials $(\mathrm{P}<$ $0.10)$, when the random effect model was chosen. Trim and fill test has been considered a conservative test [21]. So, we used a more conservative test and still found positive results for publication bias. Clinically, the heterogeneity is evident and we are going to discuss this topic ahead. It is know that random effects model for meta-analysis, although having the advantage of incorporating heterogeneity and giving large and conservative confidence intervals for the effect, this model has the disadvantage of giving more weight to small studies [22]. Influenced by small studies, when there is publication bias, meta-analysis results will became positive, when they are really neutral. It is plausible to think in this way when the two large studies with two thirds of patients of meta-analyses are neutral. Pereira and Ioannidis [23] evaluated 461 meta-analyses of clinical trials on diverse interventions. They estimated that $16-37 \%$ of the statistically significant meta-analyses are false positives.

It is important to realize that funnel plot asymmetry may have causes other than bias [20]. Another source of asymmetry arises from differences in methodological quality, as the reliability of randomization used, most often 
not detailed by smaller studies in this review. Moreover, mortality was not a primary endpoint for any of the trials included and there is inconsistent timing of antibiotic prophylaxis initiation. The statistic used to measure effect can also influence the symmetry of the results. Odds ratios overestimate the relative reduction, or increase, in risk if the event rate is high. This can lead to funnel plot asymmetry if the smaller trials were consistently conducted in patients at higher risk. In fact, while about half the population in the largest study [5] involving high-risk patients had lymphoma or solid organ tumors, in the study [24] showing the highest relative reduction with prophylaxis, increased risk of infection due to activated hematological malignancy was present in $90 \%$ of the patients. In this study, the authors state there was a statistically significant difference in mortality within 1 month of infection onset $(P<0.001)$; however, long-term survival rates were not significantly different between the groups $(P>0.05)$

Another reason for funnel plot asymmetry would be the fact of many of the selected studies that compared antibiotic prophylaxis to placebo did not present data on mortality. This observation suggests the possibility of selective reporting. Selective reporting in clinical trials occurs when outcome data are collected but not reported, and when investigators do many analyses but report only the most favorable. Published articles, as well as reviews that incorporate them, may therefore be unreliable and overestimate the benefits of an intervention. Outcome reporting bias acts in addition to the selective publication of entire studies and has widespread implications. It increases the prevalence of spurious results, and reviews of the literature will therefore tend to overestimate the effects of interventions. Finally, an asymmetrical funnel plot may arise by chance. It is important to note, however, that this will always be associated with a biased overall estimate of effect when studies are combined in a meta-analysis [13].

\section{COULD THE HETEROGENEITY OF INFECTION RISK OF PATIENTS AMONG THE STUDIES HAVE INFLUENCED THE RESULTS OF THE META- ANALYSIS?}

The mortality rate among patients with neutropenia is influenced by factors unrelated to prophylaxis, such as the response to empirical antibiotic therapy, the severity of underlying disease, and the presence of comorbidity $[25,26]$. Consequently, it is expected that the impact of antibiotic prophylaxis depends on the population studied. This is an important factor to be considered in assessing the impact of prophylaxis: if there is a benefit, which patients would benefit from it? If we assess mortality impact of quinolone prophylaxis after detachment of all trials included in metaanalysis according to the patient's risk of infection, we found no statistically significant difference between patients receiving or not quinolones prophylaxis. But it should be noted that when performing subgroup analysis of small samples we lost power to show significance. Agreeing with this, in both studies, the GIMEMA [5], that looked at a population of high-risk patients with acute leukemia and high-dose chemotherapy and the SIGNIFICANT trial [6], which addressed outpatients receiving chemotherapy for solid tumors or lymphoma (low risk patients), the reduction in mortality did not reach statistical significance. However, the current risk classification system gathers very different conditions. For example, active acute myeloid leukemia and multiple myeloma undergoing autologous HSCT are both constituents of high-risk group. Although autologous HSCT recipients also typically experience 7 days of neutropenia after conditioning, they appear to be at lower risk for serious bacterial infections [10].

\section{WHAT IS THE IMPACT OF QUINOLONE PROPHYLAXIS ON MICROBIAL RESISTANCE?}

It is well established that that use of prophylaxis can lead to greater second-line antibiotic modification and use of more potent antibiotics. Observational studies have shown that routine prophylactic use of antibiotics in neutropenic patients causes colonization of individual patients with resistant organisms [27, 28]. In a systematic review and meta-analysis done by Gafter-Gvili et al., in 2007 [29], comparing with no treatment, quinolone prophylaxis resulted in a non-significantly higher rate of resistant colonization (RR $0.49 ; 95 \%$ CI $0.37-0.66,3$ trials). Compared with trimethoprim-sulfamethoxazole, fewer quinolone-resistant bacteria were observed in the quinolone arm than trimethoprim-sulfamethoxazole-resistant bacteria in the trimethoprim-sulfamethoxazole arm (RR $0.45 ; 95 \%$ CI 0.27 $0.74,6$ trials). Furthermore, among infections developing in the two groups, quinolone-resistant infections were significantly more prevalent in the quinolone arm; $30 \%$ of all microbiologically documented infections were resistant to quinolones. All prophylactic antibiotics were associated with an increased risk for adverse events. Although further research is required to demonstrate the clinical significance of this observation, including cross-resistance to other antibiotics and survival impact, these data illustrate the importance of campaigning to limit the unnecessary use of antibiotics in situations in which evidence for benefit is lacking. At institutions that use fluoroquinolone prophylaxis, systematic monitoring of the prevalence of fluoroquinolone resistance among gram-negative bacilli is recommended.

\section{SUMMARY}

In most areas of health care, there are many studies for people involved in providing care to identify and consider when making decisions. Researchers have recognized this problem and many have accepted the challenge of preparing systematic reviews of individual studies in order to appraise summaries and bring together existing studies in a single place. Also, meta-analysis is performed when possible. In recent years however, decision makers who were once overwhelmed by the number of individual studies had to face a plethora of reviews and meta-analysis. These reviews are likely to be of variable quality and scope, with more than one systematic review on important topics. There is debate on antibiotic prophylaxis during chemotherapy. Small study effect probably exaggerated the positive results about mortality. Researchers should always check for the presence of publication bias and perform a sensitivity analysis to assess the potential impact of missing studies. Ideally, guidelines for meta-analysis publication [30] should include recommendations for authors presenting the Forest plot of studies ordered by sample size. It would be easier for readers to visualize small studies effect. 
Based on Leibovici et al., data [7], the largest metaanalysis, the average death rate in patients not receiving prophylaxis was $5 \%$, while in the group of patients receiving prophylaxis mortality was $3 \%$. This effect may be truly nonnull, but may still be an exaggerated estimate compared with the truth [31]. Accepting 2\% reduction as true, the estimated total sample size needed for a study with $80 \%$ power would be 3012 patients to find this difference in proportions. Such a large study to answer this question would have a very high cost. Systematic reviews are valuable tools for synthesizing the results of multiple and small original studies and they can also provide robust evidence. The risk of using meta-analysis instead of a large controlled randomized trial occurs when its results are influenced by small studies in the presence of publication bias. The method of combining studies with the random-effects model tends to give more weight to small studies [21]. This could explain why results of a metaanalysis including two large neutral studies, with two-thirds of patients, becomes positive.

The available evidence about quinolones prophylaxis for neutropenic patients is hypothesis generator but not sufficient for a practical clinical recommendation. It is still possible that there is a $2 \%$ absolute risk reduction in mortality given the reduction in the Leibovici et al., metaanalysis and Bucaneve et al., study. Despite possible, it has not been proved yet. A major argument against antibiotic prophylaxis is the lack of evidence of a significant reduction in mortality. On the other hand, it is necessary to discuss whether other outcomes would be useful in evaluating the cost or risk versus benefit of quinolones prophylaxis for a particular service or group of patients, such as duration of hospitalization and antibiotic use. Data regarding the time period during which mortality was assessed were scarce and varied among the trials that reported it. Clear differentiation between patients with high and low risk for infection complications is another possibility to be further explored and may be relevant in assessing the impact of antibiotic prophylaxis.

\section{ACKNOWLEDGEMENT}

Declared none.

\section{AUTHORS' CONTRIBUTIONS}

GFC, TF and MRS were involved in the conception, literature search, writing and critical revision of the manuscript. All authors have read and approved the final version of the manuscript.

\section{COMPETING INTERESTS}

The authors declare that they have no competing interests.

\section{REFERENCES}

[1] Rotstein CML, Goldberg N. Fluoroquinolone prophylaxis for profoundly neutropenic cancer patients: a meta-analysis. Curr Oncol 1997; 4 (Suppl 2): 2-7.

[2] Engels EA, Lau J, Barza M. Efficacy of quinolone prophylaxis in neutropenic cancer patients: a meta-analysis. J Clin Oncol 1998; 16(3): 1179-87.

[3] Cruciani M, Rampazzo R, Malena M, et al. Prophylaxis with fluoroquinolones for bacterial infections in neutropenic patients: a meta-analysis. Clin Infect Dis 1996; 23(4): 795-805.
[4] Van de Wetering MD, de Witte MA, Kremer LC, Offringa M, Scholten RJ, Caron HN. Efficacy of oral prophylactic antibiotics in neutropenic afebrile oncology patients: a systematic review of randomized controlled trials. Eur J Cancer 2005; 41(10): 1372-82.

[5] Bucaneve G, Micozzi A, Menichetti F, et al. Levofloxacin to prevent bacterial infection in patients with cancer and neutropenia. N Engl J Med 2005; 353(10): 977-87.

[6] Cullen M, Steven N, Billingham L, et al. Antibacterial prophylaxis after chemotherapy for solid tumors and lymphomas. N Engl J Med 2005; 353(10): 988-98.

[7] Leibovici L, Paul M, Cullen M, et al. Antibiotic prophylaxis in neutropenic patients: new evidence, practical decisions. Cancer 2006; 107(8):1743-51.

[8] Imran H, Tleyjeh IM, Arndt CA, et al. Fluoroquinolone prophylaxis in patients with neutropenia: a meta-analysis of randomized placebo-controlled trials. Eur J Clin Microbiol Infect Dis 2008; 27(1): 53-63.

[9] Gafter-Gvili A, Fraser A, Paul M, Leibovici L. Meta-analysis: antibiotic prophylaxis reduces mortality in neutropenic patients. Ann Int Med 2005; 142(12 Pt 1): 979-95.

[10] Freifeld AG, Bow EJ, Sepkowitz KA, et al. Clinical practice guideline for the use of antimicrobial agents in neutropenic patients with cancer: 2010 Update by the Infectious Diseases Society of America. Clin Infect Dis 2010; 52(4): 427-31.

[11] Tomblyn M, Chiller T, Einsele H, et al. Guidelines for preventing infectious complications among hematopoietic cell transplantation recipients: a global perspective. Biol Blood Marrow Transplant 2009; 15(10): 1143-238

[12] Gafter-Gvili A, Fraser A, Paul M, van de Wetering M, Kremer L, Leibovici L. Antibiotic prophylaxis for bacterial infections in afebrile neutropenic patients following chemotherapy. Cochrane Database Syst Rev 2005; (4): CD004386.

[13] Jonathan ACS, Sterne, Matthias E, George DS. Systematic reviews in health care: Investigating and dealing with publication and other biases in meta-analysis. BMJ 2001; 323: 14.

[14] Egger M, Dickersin K, Davey Smith G. In: Egger M, Davey Smith G, Altman DG, Eds. Systematic reviews in health care: meta-analysis in context. $2^{\text {nd }}$ ed. London: BMJ Publishing Group 2001; pp. 285-312.

[15] Jüni P, Altman DG, Egger M. Assessing the quality of controlled clinical trials. BMJ 2001; 323: 42.

[16] Sutton AJ, Duval SJ, Tweedie RL, Abrams KR, Jones DR. Empirical assessment of effect of publication bias on metaanalyses. BMJ 2000; 320(7249): 1574-7.

[17] Egger M, Smith GD. Bias in location and selection of studies. BMJ 1998; 316(7124): 61-6.

[18] Egger M, Davey Smith G, Schneider M, Minder C. Bias in metaanalysis detected by a simple, graphical test. BMJ 1997; 315(7109): 629-34.

[19] Higgins JPT, Green S, Eds. Cochrane Handbook for Systematic Reviews of Interventions Version 5.1.0 [updated March 2011]. The Cochrane Collaboration, 2011. Reference available from www.cochrane-handbook.org.

[20] Sterne JA, Sutton AJ, Ioannidis JP, et al. Recommendations for examining and interpreting funnel plot asymmetry in meta-analyses of randomized controlled trials. BMJ 2011; 343: d4002.

[21] Greenland S. Invited commentary: a critical look at some popular meta-analytic methods. Am J Epidemiol 1994; 140(3): 290-6.

[22] Schwarzer G, Carpenter J, Rücker G. Empirical evaluation suggests Copas selection model preferable to trim-and-fill method for selection bias in meta-analysis. J Clin Epidemiol 2010; 63(3): 2828.

[23] Pereira VT, Ioannidis PAJ. Statistically significant meta-analyses of clinical trials have modest credibility and inflated effects. J Clin Epidemiol 2011; 64(10): 11060-9.

[24] Nenova IS, Ananostev NH, Goranov SE, Mateva NG, Haidushka IA. Fluoroquinolone prophylaxis for bacterial infections in neutropenic patients with hematologic malignancies. Folia Med (Plovdiv) 2001; 43(4): 40-5.

[25] Bow EJ. Management of the febrile neutropenic cancer patient: lessons from 40 years of study. Clin Microbiol Infect 2005; 11 (Suppl 5): 24-9.

[26] Klastersky J, Paesmans M, Rubenstein EB, et al. The Multinational Association for Supportive Care in Cancer risk index: A multinational scoring system for identifying low-risk febrile neutropenic cancer patients. J Clin Oncol 2000; 18(16): 3038-51. 
[27] Choi SM, Park SH, Lee DG, Choi JH, Yoo JH, Shin WS. Current antimicrobial usage for the management of neutropenic fever in Korea: a nationwide survey. J Korean Med Sci 2008; 23(6): 941-7.

[28] Kern WV, Steib-Bauert M, de With K, et al. Fluoroquinolone consumption and resistance in hematology-oncology patients: ecological analysis in two university hospitals 1999-2002. J Antimicrob Chemother 2005; 55(1): 57-60.

[29] Gafter-Gvili A, Fraser A, Paul M, Leibovici L. Effect of quinolone prophylaxis in afebrile neutropenic patients on microbial resistance: systematic review and meta-analysis. J Antimicrob Chemother 2007; 59: 5-22.

[30] Liberati A, Altman DG, Tetzlaff J, et al. The PRISMA statement for reporting systematic reviews and meta-analyses of studies that evaluate healthcare interventions: explanation and elaboration. BMJ 2009; 339: b2700.

[31] Ioannidis JPA. Meta-research: The art of getting it wrong. Res Synth Methods 2010; 1(3-4): 169-84.

Received: November 2, 2011

Revised: March 7, 2012

Accepted: March 10, 2012

(C) Cota et al.; Licensee Bentham Open.

This is an open access article licensed under the terms of the Creative Commons Attribution Non-Commercial License (http://creativecommons.org/licenses/ by-nc/3.0/) which permits unrestricted, non-commercial use, distribution and reproduction in any medium, provided the work is properly cited. 